\section{Hemi-attention Syndrome}

\author{
Janna L. Harris
}

Department of Anatomy and Cell Biology, University of Kansas Medical Center, Kansas City, KS, USA

Hoglund Brain Imaging Center, University of Kansas Medical Center, Kansas City, KS, USA

\section{Synonyms}

Hemi-neglect; Unilateral neglect

\section{Definition}

Hemi-attention is the failure to respond to sensory stimuli within one hemispace after an acquired lesion to the contralateral hemisphere of the brain. Hemi-attention is observed more frequently after damage to the right hemisphere than the left, and may result from lesions within several brain regions including parietal cortex, frontal cortex, cingulate gyrus, basal ganglia, or cerebellum. A patient with hemi-attention syndrome faces significant difficulties in day-to-day life, as he/she may fail to identify objects on one side of the visual field, fail to localize sounds originating from the contralesional hemispace, fail to dress one side of his/her body, or shave one side of his face. Hemi-attention patients are often unaware that they have any deficit. For many patients the symptoms of hemi-attention syndrome resolve over the course of several months.

\section{Cross-References}

- Anosognosia

- Hemispatial Neglect

$>$ Neglect and Hemi-inattention

$\checkmark$ Neglect Syndrome

Vertical Neglect

$>$ Visual Neglect 